\title{
A Multi-Encoding Approach for LTL Symbolic Satisfiability Checking*
}

\author{
Kristin Y. Rozier $^{12 \dagger}$ and Moshe Y. Vardi ${ }^{2}$ \\ 1 NASA Ames Research Center, Moffett Field CA, 94035, USA. \\ Kristin.Y.Rozier@nasa.gov, http://ti.arc.nasa.gov/profile/kyrozier/ \\ 2 Rice University, Houston, Texas 77005, USA. \\ vardi@cs.rice.edu, http://www.cs.rice.edu/ṽardi/
}

\begin{abstract}
Formal behavioral specifications written early in the system-design process and communicated across all design phases have been shown to increase the efficiency, consistency, and quality of the system under development. To prevent introducing design or verification errors, it is crucial to test specifications for satisfiability. Our focus here is on specifications expressed in linear temporal logic (LTL).

We introduce a novel encoding of symbolic transition-based Büchi automata and a novel, "sloppy," transition encoding, both of which result in improved scalability. We also define novel BDD variable orders based on tree decomposition of formula parse trees. We describe and extensively test a new multi-encoding approach utilizing these novel encoding techniques to create 30 encoding variations. We show that our novel encodings translate to significant, sometimes exponential, improvement over the current standard encoding for symbolic LTL satisfiability checking.
\end{abstract}

\section{Introduction}

In property-based design formal properties, written in temporal logics such as LTL [31], are written early in the system-design process and communicated across all design phases to increase the efficiency, consistency, and quality of the system under development $[34,36]$. Property-based design and other design-for-verification techniques capture design intent precisely, and use formal logic properties both to guide the design process and to integrate verification into the design process [24]. The shift to specifying desired system behavior in terms of formal logic properties risks introducing specification errors in this very initial phase of system design, raising the need for property assurance [30,34].

The need for checking for errors in formal LTL properties expressing desired system behavior first arose in the context of model checking, where vacuity checking aims

\footnotetext{
* A full version of this paper with appendices is available at http://ti.arc.nasa.gov/m/profile/ kyrozier/papers/RozierVardiFM2011.pdf.

$\dagger$ Work contributing to this paper was completed at Rice University, Cambridge University, and NASA, was supported in part by the Shared University Grid at Rice (SUG@R), and was funded by NSF under Grant EIA-0216467, NASA's Airspace Systems Program, and a partnership between Rice University, Sun Microsystems, and Sigma Solutions, Inc.
} 
at reducing the likelihood that a property that is satisfied by the model under verification is an erroneous property $[2,27]$. Property assurance is more challenging at the initial phases of property-based design, before a model of the implementation has been specified. Inherent vacuity checking is a set of sanity checks that can be applied to a set of temporal properties, even before a model of the system has been developed, but many possible errors cannot be detected by inherent vacuity checking [19].

A stronger sanity check for a set of temporal properties is LTL realizability checking, in which we test whether there is an open system that satisfies all the properties in the set [32], but such a test is very expensive computationally. In LTL satisfiability checking, we test whether there is a closed system that satisfies all the properties in the set. The satisfiability test is weaker than the realizability test, but its complexity is lower; it has the same complexity as LTL model checking [39]. In fact, LTL satisfiability checking can be implemented via LTL model checking; see below.

Indeed, the need for LTL satisfiability checking is widely recognized $[14,23,25$, $28,35]$. Foremost, it serves to ensure that the behavioral description of a system is internally consistent and neither over- or under-constrained. If an LTL property is either valid, or unsatisfiable this must be due to an error. Consider, for example, the specification always $\left(b_{1} \rightarrow\right.$ eventually $\left.b_{2}\right)$, where $b_{1}$ and $b_{2}$ are propositional formulas. If $b_{2}$ is a tautology, then this property is valid. If $b_{2}$ is a contradiction, then this property is unsatisfiable. Furthermore, the collective set of properties describing a system must be satisfiable, to avoid contradictions between different requirements. Satisfiability checking is particularly important when the set of properties describing the design intent continues to evolve, as properties are added and refined, and have to be checked repeatedly. Because of the need to consider large sets of properties, it is critical that the satisfiability test be scalable, and able to handle complex temporal properties. This is challenging, as LTL satisfiability is known to be PSPACE-complete [39].

As pointed out in [35], satisfiability checking can be performed via model checking: a universal model (that is, a model that allows all possible traces) does not satisfy a linear temporal property $\neg f$ precisely when $f$ is satisfiable. In [35] we explored the effectiveness of model checkers as LTL satisfiability checkers. We compared there the performance of explicit-state and symbolic model checkers. Both use the automatatheoretic approach [43] but in a different way. Explicit-state model checkers translate LTL formulas to Büchi automata explicitly and then use an explicit graph-search algorithm [11]. For satisfiability checking, the construction of the automaton is the more demanding task. Symbolic model checkers construct symbolic encodings of automata and then use a symbolic nonemptiness test. The symbolic construction of the automaton is easy, but the nonemptiness test is computationally demanding. The extensive set of experiments described in [35] showed that the symbolic approach to LTL satisfiability is significantly superior to the explicit-state approach in terms of scalability.

In the context of explicit-state model checking, there has been extensive research on optimized construction of automata from LTL formulas [12, 13, 20-22, 38, 40, 41], where a typical goal is to minimize the size of constructed automata [42]. Optimizing the construction of symbolic automata is more difficult, as the size of the symbolic representation does not correspond directly to its optimality. An initial symbolic encoding of automata was proposed in [6], but the optimized encoding we call $C G H$, proposed 
by Clarke, Grumberg, and Hamaguchi [10], has become the de facto standard encoding. CGH encoding is used by model checkers such as CadenceSMV and NuSMV, and has been extended to symbolic encodings of industrial specification languages [9]. Surprisingly, there has been little follow-up research on this topic.

In this paper, we propose novel symbolic LTL-to-automata translations and utilize them in a new multi-encoding approach to achieve significant, sometimes exponential, improvement over the current standard encoding for LTL satisfiability checking. First we introduce and prove the correctness of a novel encoding of symbolic automata inspired by optimized constructions of explicit automata [12,22]. While the CGH encoding uses Generalized Büchi Automata (GBA), our new encoding is based on TransitionBased Büchi Automata (TGBA). Second, inspired by work on symbolic satisfiability checking for modal logic [29], we introduce here a novel sloppy encoding of symbolic automata, as opposed to the fussy encoding used in CGH. Sloppy encoding uses looser constraints, which sometimes results in smaller BDDs. The sloppy approach can be applied both to GBA-based and TGBA-based encodings, provided that one uses negationnormal form (NNF), [40], rather than the Boolean normal form (BNF) used in CGH. Finally, we introduce several new variable-ordering schemes, based on tree decomposition of the LTL parse tree, inspired by observations that relate tree decompositions to BDD variable ordering [17]. The combination of GBA/TGBA, fussy/sloppy, BNF/NNF, and different variable orders yields a space of 30 possible configurations of symbolic automata encodings. (Not all combinations yield viable configurations.)

Since the value of novel encoding techniques lies in increased scalability, we evaluate our novel encodings in the context of LTL satisfiability checking, utilizing a comprehensive and challenging collection of widely-used benchmark formulas [7, 14, 23, 35]. For each formula, we perform satisfiability checking using all 30 encodings. (We use CadenceSMV as our experimental platform.) Our results demonstrate conclusively that no encoding performs best across our large benchmark suite. Furthermore, no single approach-GBA vs. TGBA, fussy vs. sloppy, BNF vs. NNF, or any one variable order, is dominant. This is consistent with the observation made by others [1,42], that in the context of symbolic techniques one typically does not find a "winning" algorithmic configuration. In response, we developed a multi-encoding tool, PANDA, which runs several encodings in parallel, terminating when the first process returns. Our experiments demonstrate conclusively that the multi-encoding approach using the novel encodings invented in this paper achieves substantial improvement over CGH, the current standard encoding; in fact PANDA significantly bested the native LTL model checker built into CadenceSMV.

The structure of this paper is as follows. We review the CGH encoding [10] in Section 2. Next, in Section 3, we describe our novel symbolic TGBA encoding. We introduce our novel sloppy encoding and our new methods for choosing BDD variable orderings and discuss our space of symbolic encoding techniques in Section 4. After setting up our scalability experiment in Section 5, we present our test results in Section 6 , followed by a discussion in Section 7. Though our construction can be used with different symbolic model checking tools, in this paper, we follow the convention of [10] and give examples of all constructions using the SMV syntax. 


\section{Preliminaries}

We assume familiarity with LTL [16]; For convenience, Appendix A defines LTL semantics. We use two normal forms:

Definition 1 Boolean Normal Form (BNF) rewrites the input formula to use only $\neg$, $\vee, \mathcal{X}, \mathcal{U}$, and $\mathcal{F}$. In other words, we replace $\wedge, \rightarrow, \mathcal{R}$, and $\mathcal{G}$ with their equivalents:

$$
\begin{aligned}
& g_{1} \wedge g_{2} \equiv \neg\left(\neg g_{1} \vee \neg g_{2}\right) \quad g_{1} \mathcal{R} g_{2} \equiv \neg\left(\neg g_{1} \mathcal{U} \neg g_{2}\right) \\
& g_{1} \rightarrow g_{2} \equiv \neg g_{1} \vee g \quad \mathcal{G} g_{1} \equiv \neg \mathcal{F} \neg g_{1}
\end{aligned}
$$

Definition 2 Negation Normal Form (NNF) pushes negation inwards until only atomic propositions are negated, using the following rules:

$$
\begin{aligned}
\neg \neg g & \equiv g \\
\neg\left(g_{1} \wedge g_{2}\right) & \equiv\left(\neg g_{1}\right) \vee\left(\neg g_{2}\right) \\
\neg\left(g_{1} \vee g_{2}\right) & \equiv\left(\neg g_{1}\right) \wedge\left(\neg g_{2}\right) \\
\left(g_{1} \rightarrow g_{2}\right) & \equiv\left(\neg g_{1}\right) \vee g_{2}
\end{aligned}
$$

$$
\begin{aligned}
\neg(\mathcal{X} g) & \equiv \mathcal{X}(\neg g) \\
\neg\left(g_{1} \mathcal{U} g_{2}\right) & \equiv\left(\neg g_{1} \mathcal{R} \neg g_{2}\right) \\
\neg\left(g_{1} \mathcal{R} g_{2}\right) & \equiv\left(\neg g_{1} \mathcal{U} \neg g_{2}\right) \\
\neg(\mathcal{G g}) & \equiv \mathcal{F}(\neg g) \\
\neg(\mathcal{F} g) & \equiv \mathcal{G}(\neg g)
\end{aligned}
$$

In automata-theoretic model checking, we represent LTL formulas with Büchi automata.

Definition 3 A Generalized Büchi Automaton (GBA) is a quintuple ( $\left.Q, \Sigma, \delta, Q_{0}, F\right)$, where:

- $\delta \subseteq Q \times \Sigma \times Q$ is a transition relation.

- $Q$ is a finite set of states.

- $Q_{0} \subseteq Q$ is a set of initial states.

- $\Sigma$ is a finite alphabet.

- $F \subseteq 2^{Q}$ is a set of accepting state sets.

A run of a Büchi automaton A over an infinite trace $\pi=\pi_{0}, \pi_{1}, \pi_{2}, \ldots \in \Sigma$ is a sequence $q_{0}, q_{1}, q_{2}, \ldots$ of states such that $q_{0} \in Q_{0}$, and $\left\langle q_{i}, \pi_{i}, q_{i+1}\right\rangle \in \delta$ for all $i \geq 0$. A accepts $\pi$ if the run over $\pi$ visits states in every set in $F$ infinitely often. We denote the set of infinite traces accepted by $A$ by $\mathcal{L}_{\omega}(A)$.

A trace satisfying LTL formula $f$ is an infinite run over the alphabet $\Sigma=2^{\text {Prop }}$, where Prop is the underlying set of atomic propositions. We denote by models $(f)$ the set of traces satisfying $f$. The next theorem relates the expressive power of LTL to that of Büchi automata.

Theorem 1 [44] Given an LTL formula $f$, we can construct a generalized Büchi automaton $A_{f}=\left\langle Q, \Sigma, \delta, Q_{0}, F\right\rangle$ such that $|Q|$ is in $2^{O(f f \mid)}, \Sigma=2^{\text {Prop }}$, and $\mathcal{L}_{\omega}\left(A_{f}\right)$ is exactly models $(f)$.

This theorem reduces LTL satisfiability checking to automata-theoretic nonemptiness checking, as $f$ is satisfiable iff models $(f) \neq \emptyset$ iff $\mathcal{L}_{\omega}\left(A_{f}\right) \neq \emptyset$.

LTL satisfiability checking relates to LTL model checking as follows. We use a universal model $M$ that generates all traces over Prop such that $\mathcal{L}_{\omega}(M)=\left(2^{\text {Prop }}\right)^{\omega}$. The code for this model appears in [35] and Appendix B. We now have that $M$ does not satisfy $\neg f$ iff $f$ is satisfiable. We use a symbolic model checker to check the formula $\neg f$ against $M ; f$ is satisfiable precisely when the model checker finds a counterexample. 
CGH encoding In this paper we focus on LTL to symbolic Büchi automata compilation. We recap the CGH encoding [10], which assumes that the formula $f$ is in BNF, and then forms a symbolic GBA. We first define the CGH-closure of an LTL formula $f$ as the set of all subformulas of $f$ (including $f$ itself), where we also add the formula $\mathcal{X}(g \mathcal{U} h)$ for each subformula of the form $g \mathcal{U} h$. The $\mathcal{X}$-formulas in the CGH-closure of $f$ are called elementary formulas.

We declare a Boolean SMV variable $E L_{X g}$ for each elementary formula $X g$ in the CGH-closure of $f$. Also, each atomic proposition in $f$ is declared as a Boolean SMV variable. We define an auxiliary variable $S_{h}$ for every formula $h$ in the CGH-closure of $f$. (Auxiliary variables are substituted away by SMV and do not required allocated BDD variables.) The characteristic function for an auxiliary variable $S_{h}$ is defined as follows:

$$
\begin{aligned}
& S_{h}=p \text { if } p \in A P \quad S_{h}=! S_{g} \text { if } h=\neg g \quad S_{h}=S_{g 1} \mid S_{g 2} \quad \text { if } h=g_{1} \vee g_{2} \\
& S_{h}=E L_{h} \quad \text { if } h \text { is a formula } \mathcal{X} \quad S_{h}=S_{g_{2} \mid} \mid\left(S_{g 1} \& S_{\left.\mathcal{X}_{(g 1} u_{\left.g^{2}\right)}\right)}\right) \text { if } h=g_{1} \mathcal{U} g_{2}
\end{aligned}
$$

We now generate the SMV model $M_{f}$ :

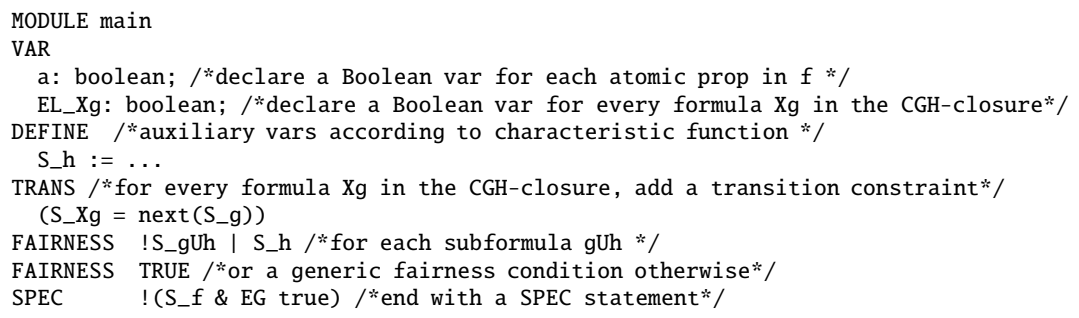

The traces of $M_{f}$ correspond to the accepting runs of $A_{f}$, starting from arbitrary states. Thus, satisfiability of $f$ corresponds to nonemptiness of $M_{f}$, starting from an initial state. We can model check such nonemptiness with SPEC ! (S_f \& EG true). A counterexample is an infinite trace starting at a state where $S_{f}$ holds. Thus, the model checker returns a counterexample that is a trace satisfying $f$.

Remark 1 While the syntax we use is shared by CadenceSMV and NuSMV, the precise semantics of CTL model checking in these model checkers is not fully documented and there are some subtle but significant differences between the two tools. Therefore, we use CadenceSMV semantics here and describe these subtleties in Appendix C.

\section{A Symbolic Transition-Based Generalized Büchi Automata (TGBA) Encoding}

We now introduce a novel symbolic encoding, referred to as TGBA, inspired by the explicit-state transition-based Generalized Büchi automata of [22]. Such automata are used by SPOT [15], which was shown experimentally [35] to be the best explicit LTL translator for satisfiability checking.

Definition 4 A Transition-Based Generalized Büchi Automaton (TGBA) is a quintuple $\left(Q, \Sigma, \delta, Q_{0}, F\right)$, where: 
- $Q$ is a finite set of states.

- $\Sigma$ is a finite alphabet.
- $\delta \subseteq Q \times \Sigma \times Q$ is a transition relation.

- $Q_{0} \subseteq Q$ is a set of initial states.

- $F \subseteq 2^{\delta}$ is a set of accepting transitions.

A run of a TGBA over an infinite trace $\pi=\pi_{0}, \pi_{1}, \pi_{2}, \ldots \in \Sigma$ is a sequence $\left\langle q_{0}, \pi_{0}, q_{1}\right\rangle$, $\left\langle q_{1}, \pi_{1}, q_{2}\right\rangle,\left\langle q_{2}, \pi_{2}, q_{3}\right\rangle$, . of transitions in $\delta$ such that $q_{0} \in Q_{0}$. The automaton accepts $\pi$ if it has a run over $\pi$ that traverses some transition from each set in $F$ infinitely often.

The next theorem relates the expressive power of LTL to that of TGBAs.

Theorem 2 [12,22] Given an LTL formula $f$, we can construct a TGBA $A_{f}=\langle Q, \Sigma, \delta$, $\left.Q_{0}, F\right\rangle$ such that $|Q|$ is in $2^{O(|f|)}, \Sigma=2^{\text {Prop }}$, and $\mathcal{L}_{\omega}\left(A_{f}\right)$ is exactly models $(f)$.

Expressing acceptance conditions in terms of transitions rather than states enables a significant reduction in the size of the automata corresponding to LTL formulas [12,22].

Our new encoding of symbolic automata, based on TGBAs, assumes that the input formula $f$ is in NNF. (This is due to the way that the satisfaction of $\mathcal{U}$-formulas is handled by means of promise variables; see below.) As in CGH, we first define the closure of an LTL formula $f$. In the case of TGBAs, however, we simply define the closure to be the set of all subformulas of $f$ (including $f$ itself). Note that, unlike in the $\mathrm{CGH}$ encoding, $\mathcal{U}$ - and $\mathcal{F}$ - formulas do not require the introduction of new $\mathcal{X}$-formulas.

The set of elementary formulas now contains: $f$; all $\mathcal{U}_{-}, \mathcal{R}_{-}, \mathcal{F}_{-}, \mathcal{G}_{-}$, and $\mathcal{G F}_{-}$ subformulas in the closure of $f$, as well as all subformulas $g$ where $\mathcal{X} g$ is in the closure of $f$. Note that we treat the common $\mathcal{G F}$ combination as a single operator.

Again, we declare a Boolean SMV variable $E L_{g}$ for every elementary formula $g$ as well as Boolean variables for each atomic proposition in $f$. In addition, we declare a Boolean SMV promise variable $P_{g}$ for every $\mathcal{U}-,(F)$-, and $\mathcal{G} \mathcal{F}$-subformula in the closure. These formulas are used to define fairness conditions. Intuitively, $P_{g}$ holds when $g$ is a promise for the future that is not yet fulfilled. If $P_{g}$ does not hold, then the promise must be fulfilled immediately. To ensure satisfaction of eventualities we require that each promise variable $P_{g}$ is false infinitely often. The TGBA encoding creates fewer $E L$ variables than the $\mathrm{CGH}$ encoding, but it does add promise variables.

Again, we define an auxiliary variable $S_{h}$ for every formula $h$ in the closure of $f$.The characteristic function for $S_{h}$ is defined as in the CGH encoding, with the following changes:

$$
\text { changes: } \begin{aligned}
S_{h}=S_{g 1} \& S_{g 2} & \text { if } h=g_{1} \wedge g_{2} \\
S_{h}=\operatorname{next}\left(E L_{g}\right) & \text { if } h=\mathcal{X} g \\
S_{h}=S_{g 2} \mid\left(S_{g 1} \& P_{g 1} \mathcal{U}_{g 2} \&\left(n \operatorname{ext}\left(E L_{g 1} \mathcal{U}_{g 2}\right)\right)\right) & \text { if } h=g_{1} \mathcal{U} g_{2} \\
S_{h}=S_{g 2} \&\left(S_{g 1} \mid\left(n \operatorname{ext}\left(E L_{g 1} \mathcal{R} g_{2}\right)\right)\right) & \text { if } h=g_{1} \mathcal{R} g_{2} \\
S_{h}=S_{g} \&\left(n \operatorname{ext}\left(E L_{\mathcal{G} g}\right)\right) & \text { if } h=\mathcal{G} g \\
S_{h}=S_{g} \mid\left(P_{\mathcal{F} g} \& n \operatorname{ext}\left(E L_{\mathcal{F}} g\right)\right) & \text { if } h=\mathcal{F} g
\end{aligned}
$$$$
S_{h}=S_{g_{2}} \&\left(S_{g 1} \mid\left(n \operatorname{ext}\left(E L_{g 1} \mathcal{R} g_{2}\right)\right)\right) \text { if } h=g_{1} \mathcal{R} g_{2}
$$$$
S_{h}=\left(\operatorname{next}\left(E L_{\mathcal{G F}} g\right)\right) \&\left(S_{g} \mid P_{\mathcal{G F}} g\right) \text { if } h=\mathcal{G F} g
$$ 
Since we reason directly over the temporal subformulas of $f$ (and not over $\mathcal{X g}$ for temporal subformula $g$ as in $\mathrm{CGH}$ ), the transition relation associates elementary formulas with matching elements of our characteristic function. Finally, we generate our symbolic TGBA; here is our SMV model $M_{f}$ :

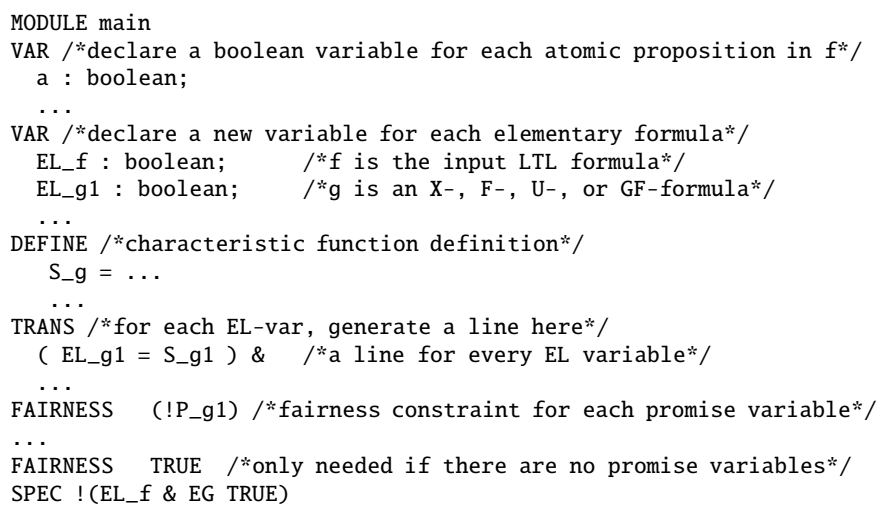

Symbolic TGBAs can only be created for NNF formulas because the model checker tries to guess a sequence of values for each of the promise variables to satisfy the subformulas, which does not work for negative $\mathcal{U}$-formulas. (This is also the case for explicit state model checking; SPOT also requires NNF for TGBA encoding [12].) Consider the formula $f=\neg(a \mathcal{U} b)$ and the trace $\mathrm{a}=1, \mathrm{~b}=0, \mathrm{a}=1, \mathrm{~b}=1, \ldots$ Clearly, $(a \mathcal{U} b)$ holds in the trace, so $f$ fails in the trace. If, however,we chose P_aUb to be false at time 0 , then EL_aUb is false at time 0 , which means that $f$ holds at time 0 . The correctness of our construction is summarized by the following theorem.

Theorem 3 Let $M_{f}$ be the SMV program made by the TGBA encoding for LTL formula $f$. Then $M_{f}$ does not satisfy the specification! (EL_f \& EG true) iff $f$ is satisfiable.

The proof of this theorem appears in Appendix D.

\section{A Set of 30 Symbolic Automata Encodings}

Our novel encodings are combinations of four components: (1) Normal Form: BNF or NNF, described above, (2) Automaton Form: GBA or TGBA, described above, (3) Transition Form: fussy or sloppy, described below, and (4) Variable Order: default, naïve, LEXP, LEXM, MCS-MIN, MCS-MAX, described below. In total, we have 30 novel encodings, since BNF can only be used with fussy-encoded GBAs, as explained below. CGH corresponds to $\mathrm{BNF} /$ fussy/GBA; we encode this combination with all six variable orders.

Automaton Form As discussed earlier, CGH is based on GBA, in combination with BNF. We can combine, however, GBA also with NNF. For this, we need to expand the characteristic function for symbolic GBAs in order to form them from NNF formulas:

$$
\begin{array}{rlrl}
S_{h}=S_{g 1} \& S_{g 2} & \text { if } h=g_{1} \wedge g_{2} & S_{h}=S_{g} \& S_{\mathcal{X}_{(\mathcal{G g})}} & \text { if } h=\mathcal{G} g \\
S_{h}=S_{g 2} \&\left(S_{g 1} \mid S_{\mathcal{X}_{(g 1 \mathcal{R} g 2)}}\right) & \text { if } h=g_{1} \mathcal{R} g_{2} & S_{h}=S_{g} \mid S_{\mathcal{X}_{(\mathcal{F g})}} \text { if } h=\mathcal{F} g
\end{array}
$$


Since our focus here is on symbolic encoding, PANDA, unlike CadenceSMV, does not apply formula rewriting and related optimizations; rather, PANDA's symbolic automata are created directly from the given normal form of the formula. Formula rewriting may lead to further improvement in PANDA's performance.

Sloppy Encoding: A Novel Transition Form CGH employs iff-transitions, of the form TRANS (EL $\mathrm{g}=\left(\mathrm{S}_{-} \mathrm{g}\right)$ ). We refer to this as fussy encoding. For formulas in NNF, we can use only-if transitions of the form TRANS (EL_g->(S-g)), which we refer to as sloppy encoding. A similar idea was shown to be useful in the context of modal satisfiability solving [29]. Sloppy encoding increases the level of non-determinism, yielding a looser, less constrained encoding of symbolic automata, which in many cases results in smaller BDDs. A side-by-side example of the differences between GBA and TGBA encodings (demonstrating the sloppy transition form) for formula $f=((\mathcal{X} a) \&(b \mathcal{U}(! a)))$ is given in Figures 1-2.

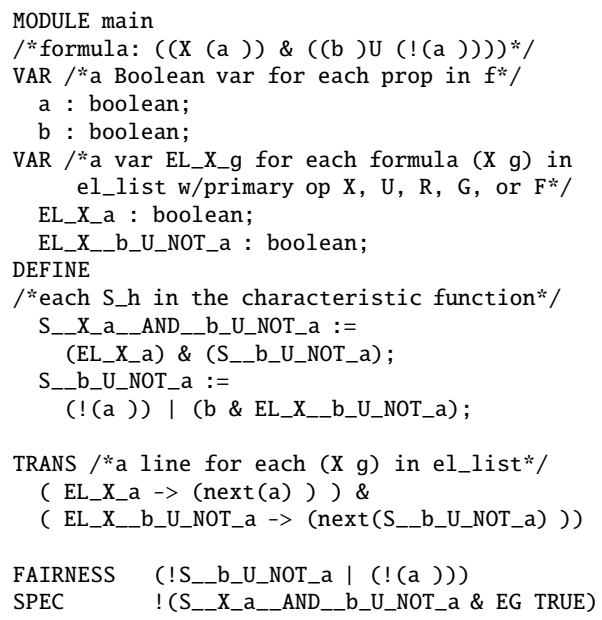

Fig. 1. NNF/sloppy/GBA encoding for CadenceSMV

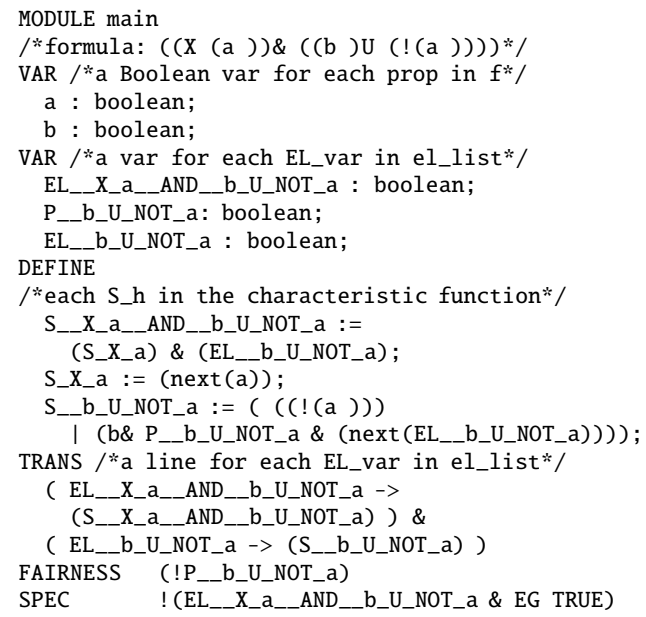

Fig. 2. NNF/sloppy/TGBA encoding for CadenceSMV

A New Way of Choosing BDD Variable Orders Symbolic model checkers search for a fair trace in the model-automaton product using a BDD-based fixpoint algorithm, a process whose efficacy is highly sensitive to variable order [5]. Finding an optimal BDD variable order is NP-hard, and good heuristics for variable ordering are crucial.

Recall that we define state variables in the symbolic model for only certain subformulas: $p \in A P, E L_{-} g$, and $P_{-} g$ for some subformulas $g$. We form the variable graph by identifying nodes in the input-formula parse tree that correspond to the primary operators of those subformulas. Since we declare different variables for the GBA and TGBA encodings, the variable graph for a formula $f$ may vary depending on the automaton form we choose. Figure 3 displays the GBA and TGBA variable graphs for an example formula, overlaid on the parse tree for this formula. We connect each variable-labeled vertex to its closest variable-labeled vertex descendant(s), skipping over vertices in the parse tree that do not correspond to state variables in our automaton construction. We 


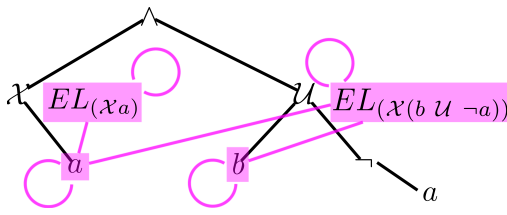

(a) GBA variable graph

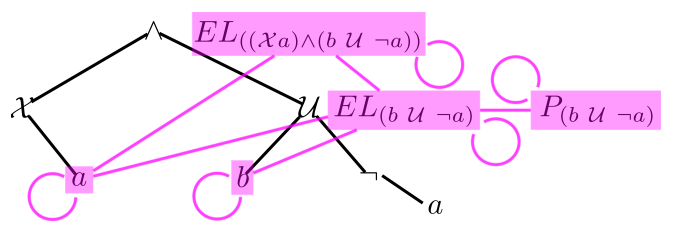

(b) TGBA variable graph

Fig. 3. Graphs in (a) and (b) were both formed from the parse tree for $f=((X a) \wedge(b \mathcal{U} \neg a))$.

create one node per subformula variable, irrespective of the number of occurrences of the subformula; for example, we create only one node for the proposition $a$ in Figure 3.

We implement five variable ordering schemes, all of which take the variable graph as input. We compare these to the default heuristic of CadenceSMV. The nä̈ve variable order is formed directly from a pre-order, depth-first trave rsal of the variable graph. We derive four additional variable-ordering heuristics by repurposing node-ordering algorithms designed for graph triangulation [26]. ${ }^{3}$ We use two variants of a lexicographic breadth-first search algorithm: variants perfect (LEXP) and minimal (LEXM). LEXP labels each vertex in the variable graph with its already-ordered neighbors; the unordered vertex with the lexicographic largest label is selected next in the variable order. LEXM operates similarly, but labels unordered vertices with both their neighbors and also all vertices that can be reached by a path of unordered vertices with smaller labels. The maximum-cardinality search (MCS) variable ordering scheme differs in the vertex selection criterion, selecting the vertex in the variable graph adjacent to the highest number of already ordered vertices next. We seed MCS with an initial vertex, chosen either to have the maximum (MCS-MAX) or minimum (MCS-MIN) degree.

\section{Experimental Methodology}

Test Methods Each test was performed in two steps. First, we applied our symbolic encodings to the input formula. Second, each symbolic automaton and variable order file pair was checked by CadenceSMV. Since encoding time is minimal and heavily dominated by model-analysis time (the time to check the model for nonemptiness to determine LTL satisfiability) we focus exclusively on the latter here.

Platform We ran all tests on Shared University Grid at Rice (SUG@R), an Intel Xeon compute cluster. ${ }^{4}$ SUG@R is comprised of 134 SunFire x4150 nodes, each with two quad-core Intel Xeon processors running at $2.83 \mathrm{GHz}$ and $16 \mathrm{~GB}$ of RAM per processor. The OS is Red Hat Enterprise 5 Linux, 2.6.18 kernel. Each test was run with exclusive access to one node. Times were measured using the Unix time command.

Input Formulas We employed a widely-used [7, 14, 23,35] collection of benchmark formulas, established by [35]. All encodings were tested using three types of scalable formulas: random, counter, and pattern. Definitions of these formulas are repeated for convenience in Appendix B. Our test set includes 4 counter and 9 pattern formula variations, each of which scales to a large number of variables, and 60,000 random formulas.

${ }^{3}$ Graph triangulation implementation coded by the Kavraki Lab at Rice University.

${ }^{4}$ http://rcsg.rice.edu/sugar/ 
Correctness In addition to proving the correctness of our algorithm, the correctness of our implementation was established by comparing for every formula in our large benchmark suite, the results (either SAT or UNSAT) returned by all encodings studied here, as well as the results returned by CadenceSMV for checking the same formula as an LTL specification for the universal model. We never encountered an inconsistency.

\section{Experimental Results}

Our experiments demonstrate that the novel encoding methods we have introduced significantly improve the translation of LTL formulas to symbolic automata, as measured in time to check the resulting automata for nonemptiness and the size of the state space we can check. No single encoding, however, consistently dominates for all types of formulas. Instead, we find that different encodings are better suited to different formulas. Therefore, we recommend using a multi-encoding approach, a variant of the multiengine approach [33], of running all encodings in parallel and terminating when the first job completes. We call our tool PANDA for "Portfolio Approach to Navigate the Design of Automata."

Seven configurations are not competitive While we can not predict the best encodings, we can reliably predict the worst. The following encodings were never optimal for any formulas in our test set. Thus, out of our 30 possible encodings, we rule out these seven:

- BNF/fussy/GBA/LEXM (essentially CGH with LEXM)

- NNF/fussy/GBA/LEXM

- NNF/fussy/TGBA/LEXM

- NNF/sloppy/GBA/LEXM
- NNF/fussy/TGBA/MCS-MAX

- NNF/sloppy/TGBA/MCS-MAX

- NNF/sloppy/TGBA/MCS-MIN

$N N F$ is the best normal form, most (but not all) of the time. NNF encodings were always better for all counter and pattern formulas; see, for example, Figure 4. Figure 5 demonstrates the use of both normal forms in the optimal encodings chosen by PANDA for random formulas. BNF encodings were occasionally significantly better than NNF; the solid point in Figure 5 corresponds to a formula for which the best BNF encoding was more than four times faster than the best NNF encoding. NNF was best much more often than BNF, likely because using NNF has the added benefit that it allows us to employ our sloppy encoding and TGBAs, which often carry their own performance advantages.

No automaton form is best. Our TGBA encodings dominated for $R 2, S$, and $U$ pattern formulas and both types of 3-variable counter formulas. For instance, the log-scale plot in Figure 6 shows that PANDA's median model analysis time for $R_{2}$ pattern formulas grows subexponentially as a function of the number of variables, while CadenceSMV's median model analysis time for the same formulas grows exponentially. (The best of PANDA's GBA encodings is also graphed for comparison.) GBA encodings are better for other pattern formulas, both types of 2-variable counter formulas, and the majority of random formulas; Figure 7 demonstrates this trend for 180 length random formulas. 

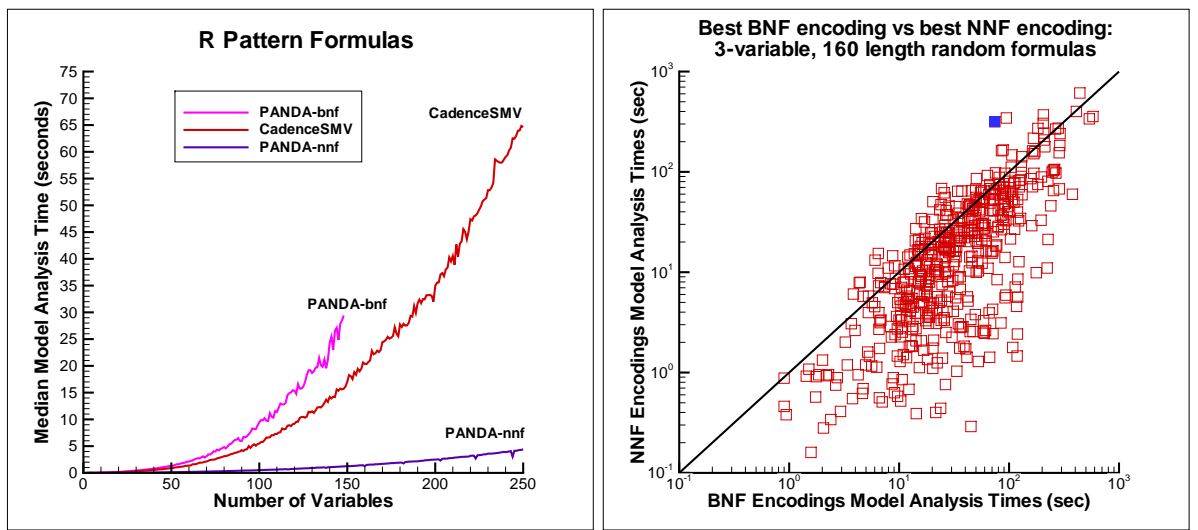

Fig. 4. Median model analysis time for Fig. 5. Best encodings of 500 3-variable, 160 $R(n)=\bigwedge_{i=1}^{n}\left(\mathcal{G F} p_{i} \vee \mathcal{F} \mathcal{G} p_{i+1}\right)$ for PANDA length random formulas. Points fall below the $\mathrm{NNF} /$ sloppy/GBA/naïve, CadenceSMV, and diagonal when NNF is better. the best BNF encoding.
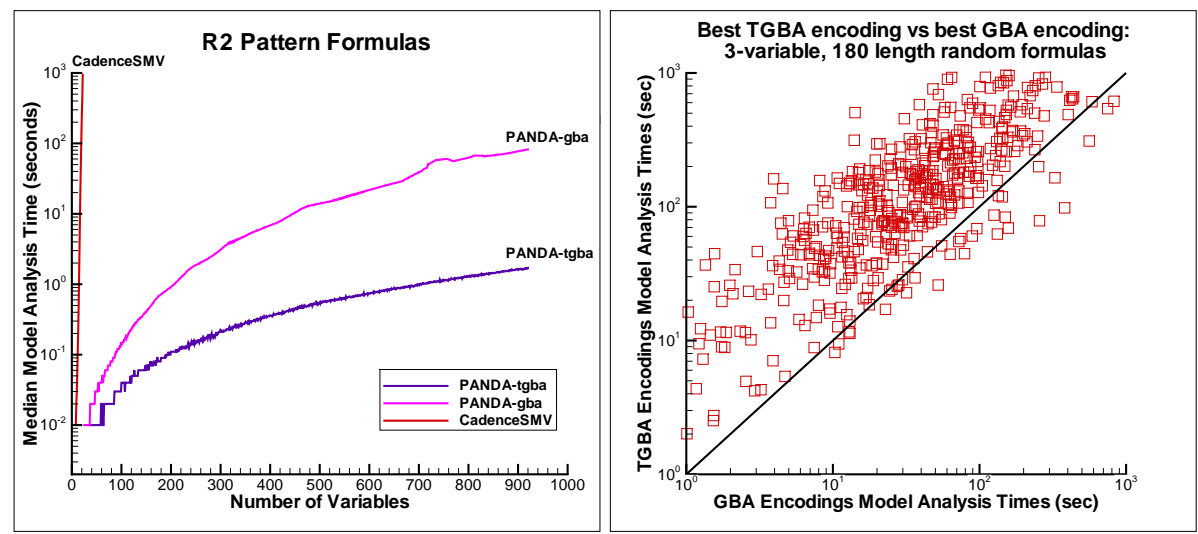

Fig. 6. $R_{2}(n)=\left(. .\left(p_{1} \mathcal{R} p_{2}\right) \mathcal{R} \ldots\right) \mathcal{R} p_{n}$. Fig. 7. Best encodings of 5003 -variable, 180 PANDA's NNF/sloppy/TGBA/LEXP encoding length random formulas.

scales better than the best GBA encoding, NNF/sloppy/GBA/naïve, and exponentially better than CadenceSMV.

No transition form is best Sloppy is the best transition form for all pattern formulas. For instance, the log-scale plot of Figure 8 illustrates that PANDA's median model analysis time for $U$ pattern formulas grows subexponentially as a function of the number of variables, while CadenceSMV's median model analysis time for the same formulas grows exponentially. Fussy encoding is better for all counter formulas. The best encodings of random formulas were split between fussy and sloppy. Figure 9 demonstrates this trend for 140 length random formulas.

No variable order is best, but LEXM is worst. The best encodings for our benchmark formula set were split between five variable orders. The naïve and default orders proved 

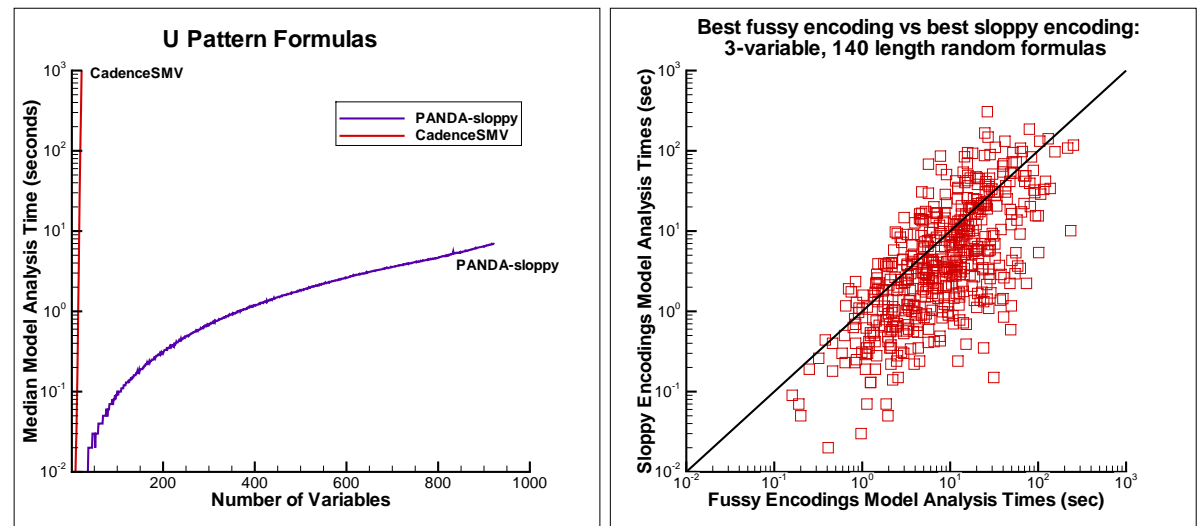

Fig. 8. $U(n)=\left(\ldots\left(p_{1} \mathcal{U} p_{2}\right) \mathcal{U} \ldots\right) \mathcal{U} p_{n}$. Fig. 9. Best encodings of 5003 -variable, 140 PANDA's NNF/sloppy/TGBA/LEXP scalables length random formulas. Points fall below the exponentially better than CadenceSMV. diagonal when sloppy encoding is best.

optimal for more random formulas than the other orders. Figure 10 demonstrates that neither the naïve order nor the default order is better than the other for random formulas. The naïve order was optimal for $E, Q, R, U_{2}$, and $S$ patterns. MCS-MAX is optimal for 2and 3-variable linear counters. The LEXP variable order dominated for $C_{1}, C_{2}, U$, and $R_{2}$ pattern formulas, as well as for 2- and 3-variable counter formulas, yet it was rarely best for random formulas. Figure 11 demonstrates the marked difference in scalability provided by using the LEXP order over running CadenceSMV on 3-variable counter formulas; We can analyze much larger models with PANDA using LEXP than with the native CadenceSMV encoding before memory-out. We never found the LEXM order to be the single best encoding for any formula.
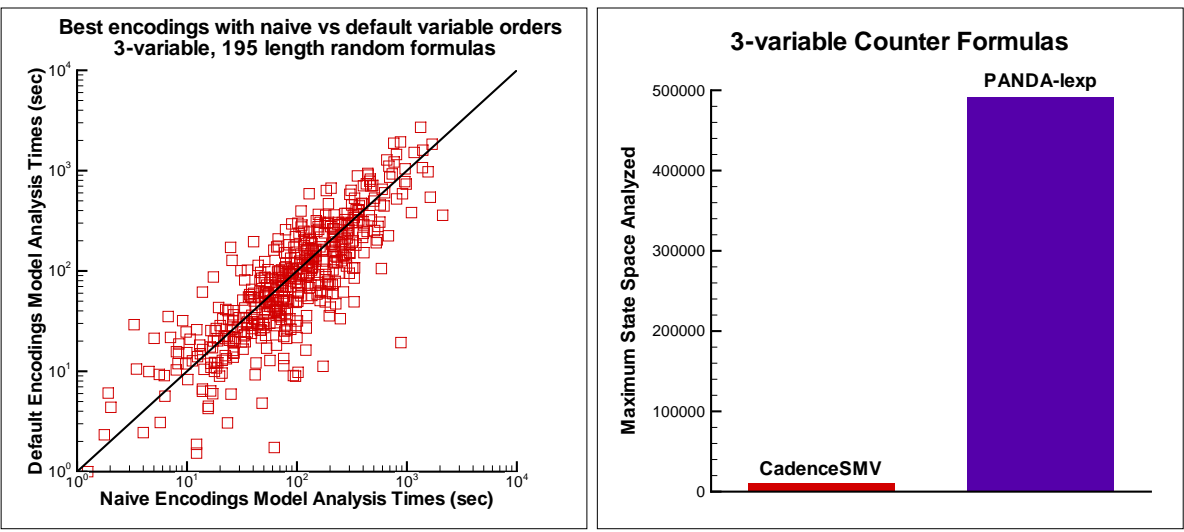

Fig. 10. Best encodings of 500 3-variable, 195 Fig. 11. Maximum states analyzed before length random formulas. Points fall above the space-out. CadenceSMV quits at 10240 states. diagonal when naïve variable order is best.

PANDA's NNF/fussy/TGBA/LEXP scales to 491520 states. 
A formula class typically has a best encoding, but predictions are difficult While each of our pattern and counter formulas had a best (or a pair of best) encodings, which remained consistent as we scaled the formulas, we found that we could not reliably predict the best encoding using any statistics gathered from parsing, such as operator counts or ratios. For example, we found that the best encoding for a pattern formula was not necessarily the best for a randomly-generated formula comprised of the same temporal operators. We surmise that the best encoding is tied to the structure of the formula on a deeper level; developing an accurate heuristic is left to future work.

There is no single best encoding; a multi-encoding approach is clearly superior We implement a novel multi-encoding approach: our new PANDA tool creates several encodings of a formula and uses a symbolic model checker to check them for satisfiability in parallel, terminating when the first check completes. Our experimental data supports this multi-encoding approach. Figures 4,6 , and 8 highlight the significant decrease in CadenceSMV model analysis time for $R, R_{2}$, and $U$ pattern formulas, while Figure 11 demonstrates increased scalability in terms of state space using counter formulas. Altogether, we demonstrate that a multi-encoding approach is dramatically more scalable than the current state-of-the-art. The increase in scalability is dependant on the specific formula, though for some formulas PANDA's model analysis time is exponentially better than CadenceSMV's model analysis time for the same class of formulas.

\section{Discussion}

This paper brought attention to the issue of scalable construction of symbolic automata for LTL formulas in the context of LTL satisfiability checking. We defined novel encodings and novel BDD variable orders for accomplishing this task. We explored the impact of these encodings, comprised of combinations of normal forms, automaton forms, transition forms, and combined with variable orders. We showed that each can have a significant impact on performance. At the same time, we showed that no single encoding outperforms all others and showed that a multi-encoding approach yields the best result, consistently outperforming the native translation of CadenceSMV.

We do not claim to have exhaustively covered the space of possible encodings of symbolic automata. Several papers on the automata-theoretic approach to LTL describe approaches that could be turned into alternative encodings of symbolic automata, cf. $[4,18,20,37]$. The advantage of the multi-encoding approach we introduced here is its extensibility; adding additional encodings is straightforward. The multi-encoding approach can also be combined with different back ends. In this paper we used CadenceSMV as a BDD-based back end; using another symbolic back end (cf. [14]) or a SAT-based back end (cf. [3]) would be an alternative approach, as both BDD-based and SAT-based back ends require symbolic automata. Since LTL serves as the basis for industrial languages such as PSL and SVA, the encoding techniques studied here may also serve as the basis for novel encodings of such languages, cf. [8,9].

In this paper we examined our novel symbolic encodings of LTL in the context of satisfiability checking. An important difference between satisfiability checking and model checking is that in the former we expect to have to handle much larger formulas, 
since we need to consider the conjunction of properties. Also, in model checking the size of the symbolic automata can be dwarfed by the size of the model under verification. Thus, the issue of symbolic encoding of automata in the context of model checking deserves a separate investigation.

\section{References}

1. N. Amla, X. Du, A. Kuehlmann, R.P. Kurshan, and K.L. McMillan. An analysis of SATbased model checking techniques in an industrial environment. In IFIG Adv. Research Working Conf. on Correct Hardware Design and Verification Methods, LNCS 3725, pages 254-268. Springer, 2005.

2. I. Beer, S. Ben-David, C. Eisner, and Y. Rodeh. Efficient detection of vacuity in ACTL formulas. FMSD 18, (2):141-162, 2001.

3. A. Biere, C. Artho, and V. Schuppan. Liveness checking as safety checking. In FMICS 66:2, ENTCS, 2002.

4. R. Bloem, A. Cimatti, I. Pill, and M. Roveri. Symbolic implementation of alternating automata. IJFCS 18, (4):727-743, 2007.

5. R.E. Bryant. Graph-based algorithms for Boolean-function manipulation. IEEE TC C-35, (8):677-691, 1986.

6. J.R. Burch, E.M. Clarke, K.L. McMillan, D.L. Dill, and L.J. Hwang. Symbolic model checking: $10^{20}$ states and beyond. Inform. and Computation 98, (2):142-170, Jun 1992.

7. J. Cichon, A. Czubak, and A. Jasinski. Minimal Büchi automata for certain classes of LTL formulas. DepCoS 0, pages 17-24, 2009.

8. A. Cimatti, M. Roveri, S. Semprini, and S. Tonetta. From PSL to NBA: A modular symbolic encoding. In FMCAD, 2006.

9. A. Cimatti, M. Roveri, and S. Tonetta. Syntactic optimizations for PSL verification. In TACAS, pages 505-518, 2007.

10. E. M. Clarke, O. Grumberg, and K. Hamaguchi. Another look at LTL model checking. Formal Methods in System Design 10, (1):47-71, 1997.

11. C. Courcoubetis, M.Y. Vardi, P. Wolper, and M. Yannakakis. Memory efficient algorithms for the verification of temporal properties. In CAV, LNCS 531, p233-242. Springer, 1990.

12. J-M. Couvreur. On-the-fly verification of Linear Temporal Logic. In FM, p253-271, 1999.

13. N. Daniele, F. Guinchiglia, and M.Y. Vardi. Improved automata generation for Linear Temporal Logic. In $C A V$, LNCS 1633, pages 249-260. Springer, 1999.

14. M. De Wulf, L. Doyen, N. Maquet, and J. Raskin. Antichains: Alternative algorithms for LTL satisfiability and model-checking. In TACAS, pages 63-77, 2008.

15. A. Duret-Lutz and D. Poitrenaud. SPOT: An extensible model checking library using Transition-Based Generalized Büchi Automata. In MASCOTS, pages 76-83, 2004.

16. E.A. Emerson. Temporal and modal logic. In Handbook of Theoretical Computer Science, volume B, chapter 16, pages 997-1072. Elsevier, MIT Press, 1990.

17. A. Ferrara, G. Pan, and M. Y. Vardi. Treewidth in verification: Local vs. global. In LPAR, LNCS 3835, pages 489-503. Springer, 2005.

18. M. Fisher. A normal form for temporal logics and its applications in theorem-proving and execution. J. Log. Comput. 7, (4):429-456, 1997.

19. D. Fisman, O. Kupferman, S. Sheinvald-Faragy, and M.Y. Vardi. A framework for inherent vacuity. In Haifa Verification Conference, LNCS 5394, pages 7-22. Springer, 2008.

20. P. Gastin and D. Oddoux. Fast LTL to Büchi automata translation. In CAV, LNCS 2102, pages 53-65. Springer, 2001. 
21. R. Gerth, D. Peled, M.Y. Vardi, and P. Wolper. Simple on-the-fly automatic verification of Linear Temporal Logic. In PSTV, pages 3-18. Chapman \& Hall, Aug 1995.

22. D. Giannakopoulou and F. Lerda. From states to transitions: Improving translation of LTL formulae to Büchi automata. In FORTE, Nov 2002.

23. V. Goranko, A. Kyrilov, and D. Shkatov. Tableau tool for testing satisfiability in LTL: Implementation and experimental analysis. ENTCS 262, pages 113-125, 2010.

24. A. Habibi and S. Tahar. Design for verification of SystemC transaction level models. In Design, Automation and Test in Europe, pages 560-565. IEEE, 2005.

25. Y. Kesten, Z. Manna, H. McGuire, and A. Pnueli. A decision algorithm for full propositional temporal logic. In $C A V$, LNCS 697, pages 97-109. Springer, 1993.

26. A. M. C. A. Koster, H. L. Bodlaender, and S. P. M. van Hoesel. Treewidth: Computational experiments. ZIB-Report 01-38, ZIB, 2001.

27. O. Kupferman and M.Y. Vardi. Vacuity detection in temporal model checking. STTT 4, (2):224-233, Feb 2003.

28. S. Merz and A. Sezgin. Emptiness of Linear Weak Alternating Automata. Technical report, LORIA, December 2003.

29. G. Pan, U. Sattler, and M.Y. Vardi. BDD-based decision procedures for K. In CADE, LNCS 2392, pages 16-30. Springer, 2002.

30. I. Pill, S. Semprini, R. Cavada, M. Roveri, R. Bloem, and A. Cimatti. Formal analysis of hardware requirements. In $D A C$, pages 821-826. ACM, 2006.

31. A. Pnueli. The temporal logic of programs. In IEEE FOCS, pages 46-57, 1977.

32. A. Pnueli and R. Rosner. On the synthesis of a reactive module. In POPL, p179-190, 1989.

33. L. Pulina and A. Tacchella. A self-adaptive multi-engine solver for quantified Boolean formulas. Constraints 14, (1):80-116, 2009.

34. M. Roveri. Novel techniques for property assurance. Technical report, PROSYD deliverable 1.2/2, 2004.

35. K.Y. Rozier and M.Y. Vardi. LTL satisfiability checking. In Model Checking Software (SPIN), LNCS 4595, pages 149-167. Springer, 2007.

36. S. Ruah, A. Fedeli, C. Eisner, and M. Moulin. Property-driven specification of VLSI design. Technical report, PROSYD deliverable 1.1/1, 2005.

37. K. Schneider. Improving automata generation for Linear Temporal Logic by considering the automaton hierarchy. In LPAR, pages 39-54. Springer, 2001.

38. R. Sebastiani and S. Tonetta. "More deterministic" vs. "smaller" Büchi automata for efficient LTL model checking. In CHARME, pages 126-140. Springer, 2003.

39. A.P. Sistla and E.M. Clarke. The complexity of Propositional Linear Temporal Logic. J. ACM 32, pages 733-749, 1985.

40. F. Somenzi and R. Bloem. Efficient Büchi automata from LTL formulae. In $C A V$, LNCS 1855, pages 248-263. Springer, 2000.

41. X. Thirioux. Simple and efficient translation from LTL formulas to Büchi automata. ENTCS 66, (2):145-159, 2002.

42. M.Y. Vardi. Automata-theoretic model checking revisited. In VMCAI, LNCS 4349, pages 137-150. Springer, 2007.

43. M.Y. Vardi and P. Wolper. An automata-theoretic approach to automatic program verification. In LICS, pages 332-344, Cambridge, Jun 1986.

44. M.Y. Vardi and P. Wolper. Reasoning about infinite computations. Information and Computation 115, (1):1-37, Nov 1994. 